\title{
Broadband Zero-Bias RF Field-Effect Rectifiers Based on AlGaN/GaN Nanowires
}

\author{
Giovanni Santoruvo, Mohammad Samizadeh Nikoo, and Elison Matioli, Member, IEEE
}

\begin{abstract}
Microwave zero-bias rectifiers are fast devices capable of rectifying RF signals without applied bias, which have applications ranging from $\mathrm{RF}$ power detection to $\mathrm{THz}$ imaging systems. Here we present gated nanowire field-effect rectifiers (NW-FERs) fabricated with a process compatible with other RF devices on a standard AlGaN-GaN High Electron Mobility Transistor (HEMT) platform as a new potential RF zero-bias diode. Signal rectification relies on the electrostatic modulation of the gated-NW carrier concentration, which is optimized by a judicious NW width design. NW-FERs presented a large curvature $\left(30.1 \mathrm{~V}^{-1}\right)$, close to the theoretical limit $\left(38.7 \mathrm{~V}^{-1}\right)$ for ideal Schottky diodes, and an excellent trade-off between a flat frequency response, up to a few tens of $\mathrm{GHz}$, and a large responsivity $(3000 \mathrm{~V} / \mathrm{W})$. The compatible fabrication process and the very good results provide a promising high-performance zerobias diode architecture that could be integrated on AlGaN/GaN microwave monolithic integrated circuits (MMICs).
\end{abstract}

Index Terms-Nanowire, RF zero-bias diode, THz imaging, RF power detection.

\section{INTRODUCTION}

G AN-based high electron mobility transistors (HEMTs) are one of the most promising platforms for the development of MMICs, for which the integration of microwave power detectors could enable both an improved performance as well as new applications. Microwave power detectors are devices generating a DC voltage proportional to the RF signal power at their input. Applications include incoherent demodulation by envelope detection, demodulation based on six-port receivers [1]-[3], terahertz imaging [4]-[6] and power monitoring to improve the efficiency of power amplifiers[7]-[9].

Detectors operating without the need for DC bias, called zero-bias detectors, are very favorable for these applications since they ensure a sharp reduction of the shot and flicker (1/f) noise, and do not require energy nor DC biasing networks to operate [10].

Excellent RF detection performances have been reached with optimized Schottky contacts on heavily engineered epitaxial structures based on $\mathrm{n} / \mathrm{n}^{+}$-GaAs [11], [12], as well as in Sb-based tunnel junctions [13]-[18], however they are not compatible with current MMIC epitaxial structures.

Zero-bias detectors based on AlGaN/GaN HEMTs have been demonstrated using nanoscale devices, such as selfswitching diodes (SSDs) presenting very high-frequency operation (up to hundreds of GHz) [19]-[21], as well as in lateral

This work was supported by the Swiss National Science Foundation under Grant 200021_169362. The authors are with the Power and Wideband-gap Electronics Research Laboratory (POWERlab), Ecole Polytechnique Federale de Lausanne (EPFL) , CH1015, Lausanne, Switzerland (e-mail: giovanni.santoruvo@epfl.ch; elison.matioli@epfl.ch) field-effect diodes (L-FEDs) showing excellent detection capabilities (responsivity in the order of thousands of $\mathrm{V}^{-1}$ ) [10], [22]-[24]. Devices based on GaN HEMT structures presenting concurrently good detection and frequency capabilities would expand the portfolio of RF devices for GaN MMICs. In this letter, we demonstrate zero-bias NW-FERs, based on an array of tri-gated nanowire HEMTs (Fig. 1a-d), presenting excellent zero-bias rectification performance. Zero-bias fieldeffect rectifiers (Fig. 1b) require the channel threshold voltage $\left(V_{\text {th }}\right)$ to be positive and close to $0 \mathrm{~V}$ [22], [25]. By using NWs as channel (Fig. 1c), $V_{\text {th }}$ could be finely tuned with high precision and reproducibility to obtain a $V_{\text {on }}$ close to $0 \mathrm{~V}$. The tri-gates resulted in a steep sub-threshold slope, which was exploited to enhance the rectification capabilities of the devices [26], obtaining detection performance approaching the behavior of ideal field effect rectifiers and Schottky diodes used as RF detectors [27].

NW-FERs are first analyzed in DC, highlighting the influence of $V_{\mathrm{TH}}$ on DC figures of merit. Then, responsivity measurements, both versus input power $\left(P_{\text {in }}\right)$ and frequency $(f)$, are shown and discussed. Finally, S-parameters are reported together with the equivalent circuit model.
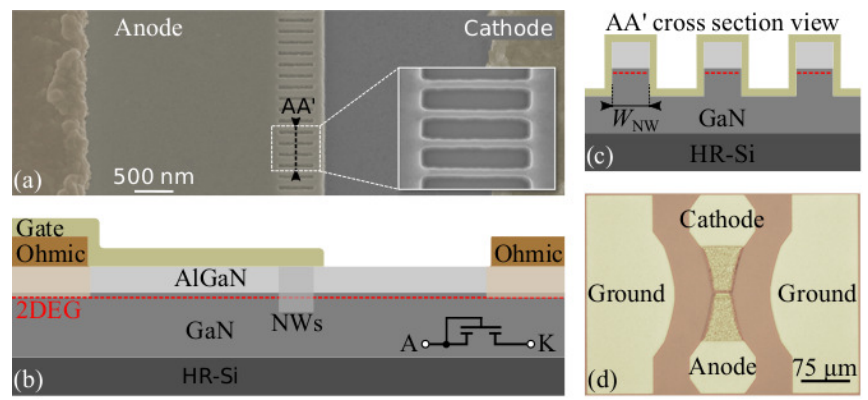

Fig. 1. (a) SEM image of a $30 \mathrm{~nm}$-wide NW-FER. The inset shows the NW array before gate metal deposition. (b) Lateral and (c) cross-section (along AA') schematics of the device. (d) Optical image of a NW-FER showing the ground-signal-ground (GSG) pads for RF measurements.

\section{RESULTS AND DISCUSSION}

NW-FERs were fabricated on $\mathrm{GaN}(2.7 \mathrm{~nm}) / \mathrm{AlGaN}$ $(25 \mathrm{~nm}) / \mathrm{GaN}(500 \mathrm{~nm})$ followed by a $1.7 \mu \mathrm{m}$-thick buffer on high-resistive silicon substrate. The electron mobility $\left(\mu_{e}\right)$, carrier concentration $\left(n_{s}\right)$ and sheet resistance $\left(R_{\mathrm{sh}}\right)$ of the epitaxial structure measured at $300 \mathrm{~K}$ were 1917 $\mathrm{cm}^{2} / \mathrm{Vs}, 1.06 \times 10^{13} \mathrm{~cm}^{-2}$ and $300 \Omega / \square$, respectively. NW-FERs were fabricated by electron-beam lithography using hydrogen silsesquioxane $2 \%$ (HSQ), followed by $\mathrm{Cl}_{2}$-based dry-etching

(C) 2019 IEEE. Personal use of this material is permitted. Permission from IEEE must be obtained for all other uses, in any current or future media, including reprinting/republishing this material for advertising or promotional purposes, creating new collective works, for resale or redistribution to servers or lists, or reuse of any copyrighted component of this work in other works. 


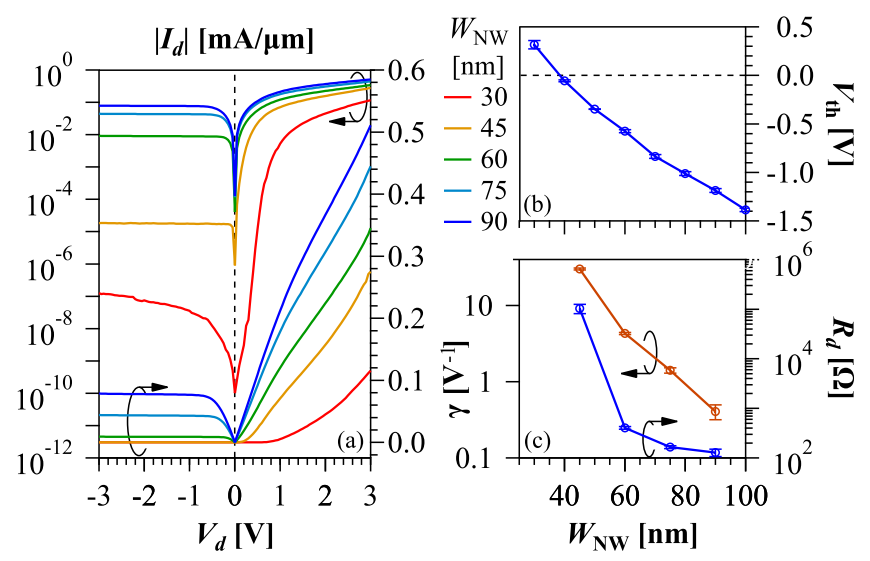

Fig. 2. (a) Diode current $\left|I_{\mathrm{d}}\right|$ versus voltage $V_{\mathrm{d}}$ in logarithmic (left axis) and linear (right axis) scales for NW-FERs with NW widths of $30 \mathrm{~nm}, 45 \mathrm{~nm}$, $60 \mathrm{~nm}, 75 \mathrm{~nm}$ and $90 \mathrm{~nm}$. (b) $V_{\text {th }}$ (defined at $1 \mu \mathrm{A} / \mathrm{mm}$ ) for Schottky tri-gate HEMTs versus NW width. (c) $\gamma$ and $R_{\mathrm{d}}$ at $V_{\mathrm{d}}=0 \mathrm{~V}$ for NW-FER with NW width from $45 \mathrm{~nm}$ to $90 \mathrm{~nm}$ (devices with $30 \mathrm{~nm}$ wide NWs are not shown since they cannot work as zero bias diode due to the large $V_{\mathrm{on}}$ ).

of the nanowires and mesa isolation. Ohmic contacts were formed by Ti (200 ̊)/Al (1200 ̊)/Ti $(400 \AA) \mathrm{Ni}(600 \AA) / \mathrm{Au}$ $(500 \AA)$ annealed at $780{ }^{\circ} \mathrm{C}$, resulting in a contact resistance of $0.81 \Omega \mathrm{mm}$, and $\mathrm{Ni}(300 \AA) / \mathrm{Au}(1300 \AA)$ was used as gate for NW arrays.

Fig. 2a shows the DC performance of NW-FERs, composed of $240 \mathrm{NWs}$ with a length of $500 \mathrm{~nm}$, widths $\left(\mathrm{W}_{\mathrm{NW}}\right)$ varying from $30 \mathrm{~nm}$ to $90 \mathrm{~nm}$ and a constant NW spacing of $100 \mathrm{~nm}$, which corresponds to a variation of the NW filling factor from $23 \%$ to $47 \%$. The device current was normalized by the width of the entire device footprint (not by the NW width).

The NW width plays a key role in defining both the $V_{\text {on }}$ and the reverse leakage current $\left(I_{\mathrm{r}}\right)$. Narrow NWs $(30 \mathrm{~nm}$ wide) presented a much reduced $I_{\mathrm{r}}$, down to $100 \mathrm{nA} / \mathrm{mm}$, but with large $V_{\text {on }}$ of around $0.4 \pm 0.05 \mathrm{~V}$ (at $1 \mathrm{~mA} / \mathrm{mm}$ ), while wider nanowires ( $90 \mathrm{~nm}$-wide) presented $V_{\text {on }}$ close to $0 \mathrm{~V}$ but with much larger leakage current, on the order of the forward current, which significantly degraded the device rectification capability. A good compromise was achieved for devices with $45 \mathrm{~nm}$-wide NWs, with $V_{\text {on }}$ of $\sim 0 \mathrm{~V}$ and a flat $I_{\mathrm{r}}$ of $20 \mu \mathrm{A} / \mathrm{mm}$.

Both $V_{\text {on }}$ and $I_{\mathrm{r}}$ are directly related to the $V_{\text {th }}$ of the NWs, which can be easily tuned from $-1.2 \mathrm{~V}$ to $+0.4 \mathrm{~V}$ by varying the NW width from $90 \mathrm{~nm}$ to $30 \mathrm{~nm}$ (Fig. 2b). Such dependence is produced by the sidewall depletion of carriers [28], strain relaxation of the barrier layer in narrow NWs [29] (which depends also on the NW length [30]) and the enhanced gate control from the tri-gate structures [26].

$V_{\text {th }}$ has also a strong impact on the device detection capability, which is characterized by the current curvature $\gamma=d^{2} I_{\mathrm{d}} / d V_{\mathrm{d}}^{2}\left(d I_{\mathrm{d}} / d V_{\mathrm{d}}\right)^{-1}$ [16], and by the differential resistance $R_{\mathrm{d}}$, both at $0 \mathrm{~V}$. For RF power detectors, low values of $R_{\mathrm{d}}$ are desirable for low thermal noise, along with high values of $\gamma$ for good power detection. Fig. 2c shows the tradeoff between $\gamma$ and $R_{\mathrm{d}}$, which are both heavily influenced by $W_{\mathrm{NW}}$. While the $45 \mathrm{~nm}$-wide NW-FERs show the largest $\gamma$ of $30.1 \pm 0.9 \mathrm{~V}^{-1}$, their high $R_{\mathrm{d}}$ can be reduced by increasing the number of NWs and by fine tuning $W_{\mathrm{NW}}$ using wet etch treatments [31], [32]. For instance, $R_{\mathrm{d}}$ was reduced by a factor of 5-6x when increasing the number of NWs from 120 to 240 and a few $\mathrm{nm}$ of $W_{\mathrm{NW}}$.

For $V_{\text {th }} \ll 0 \mathrm{~V}$ values (large $W_{\mathrm{NW}}$ ), devices work in the triode-region (high conductive region allowing small $R_{\mathrm{d}}$ ) where the current can be modeled as $I_{\mathrm{d}} \propto\left(V_{\mathrm{d}} / 2-V_{\mathrm{th}}\right) V_{\mathrm{d}}$, so $\gamma$ is equal to $-V_{\mathrm{th}}^{-1}$, which leads to a maximum $\gamma$ of a few $\mathrm{V}^{-1}$ [33]. When $V_{\text {th }}$ approaches $0 \mathrm{~V}$, devices work in the subthreshold region (low conductive region) and the current can be modeled as $I_{\mathrm{d}}^{\text {sub-th }} \propto \exp \left(-q \frac{V_{\mathrm{th}}}{k_{\mathrm{B}} T}\right)\left(\exp \left(\frac{q V_{\mathrm{d}}}{k_{\mathrm{b}} T}\right)-1\right)$, where $q$ is the elementary charge, $k_{\mathrm{b}}$ is the Boltzmann constant and $T$ is the absolute temperature, resulting in a maximum $\gamma$ of $q / k_{\mathrm{b}} T=38.7 \mathrm{~V}^{-1}$ [33]. This result resembles Schottky diodes ( $\gamma=q / n k_{\mathrm{b}} T$ for which $n$ is the ideality factor), so $45 \mathrm{~nm}$ wide NW-FERs have $\gamma$ close to the theoretical limit, thanks to the strong channel control of the tri-gate, and they behave similarly to Schottky diodes with $n=1.28$. Larger values of $\gamma$ can be achieved by exploiting different physical phenomena than field effect or Schottky barrier rectification, as in tunnel backward diodes [16].

Fig. 3a shows the rectified $V_{\text {out }}$ versus $P_{\text {in }}$ at $1 \mathrm{GHz}$ for all devices. The $30 \mathrm{~nm}$-wide NW-FERs could not detect signals below $-10 \mathrm{dBm}$ due to their large $V_{\text {on }}$, thus they are not considered for further analysis. The measurement setup used for the responsivity measurement is shown in the inset of Fig. 3.a. The reported $P_{\text {in }}$ refers to the power set in the vector network analyzer (VNA) after removing the insertion losses $(I L)$ from the input cable $\left(P_{\text {in }}=P_{\text {in }}^{\mathrm{VNA}}-I L_{\text {cable }}\right)$. $V_{\text {out }}$ has a linear behavior for a large range of $P_{\text {in }}$, in agreement with the "square-law" of diodes $\left(V_{\text {out }} \propto V_{\text {in }}^{2} \propto P_{\text {in }}\right)$ [34]. At larger $P_{\text {in }}$ the impedance of diodes starts to depend on $P_{\text {in }}$ causing the sub-linear behavior observable in Fig. 3a [34]. The minimum detectable power generating a stable $V_{\text {out }}$, measured using a multimeter (which represents a real application case), was $50 \mathrm{dBm}$ for $45 \mathrm{~nm}$-wide NW-FERs, which was degraded for wider NWs devices. The dynamic range was about $30 \mathrm{dBm}$ (calculated from the $1 \mathrm{~dB}$ compression point) independently from the NW widths.

Fig. 3b shows $\beta$, defined as $V_{\text {out }} / P_{\text {in }}$, for all devices versus $P_{\text {in }}$, revealing a significant increase of up to 2 orders of magnitude for narrow NW-FERs, which is a consequence of the much larger $\gamma$ (Fig. 2a). The 45 nm-wide NW-FERs presented a maximum $\beta$ of $3000 \mathrm{~V} / \mathrm{W}$, which is very close to the theoretical value of $2 Z_{\mathrm{s}} \gamma=3010 \mathrm{~V} / \mathrm{W}$, defined in the case of complete mismatch between the device impedance $(\sim$ $100 \mathrm{k} \Omega$ ) and the source impedance $Z_{\mathrm{s}}(50 \Omega$ of the VNA port) [33]. At large power, the responsivity approaches the theoretical value of $2 \sqrt{2 Z_{0} / P_{\text {in }}}$, which considers a capacitive load connected to an ideal diode in off-state $\left(I_{\mathrm{r}}=0 \mathrm{~A}\right)$, for which the entire reflected power causes the doubling of input voltage. Therefore, NW-FERs provide good responsivities, in the same order $(>1000 \mathrm{~V} / \mathrm{W})$ of those for state of the art devices [10], [12], [14]-[16], [35], on a platform compatible to RF GaN HEMTs. Such results were achieved without any matching network, allowing a simpler and more compact design as well as broader band operation.

Fig. 4a shows the measured frequency response of $\beta$ at $P_{\text {in }}=-20 \mathrm{dBm}$ (square law region for all devices) for a large 


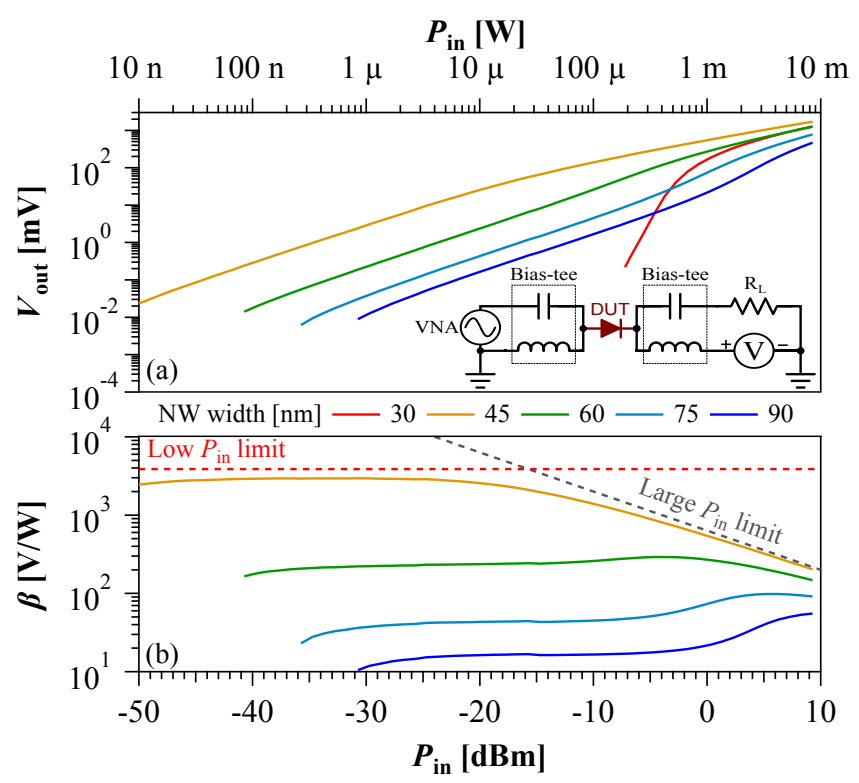

Fig. 3. (a) $V_{\text {out }}$ and (b) $\beta$ versus $P_{\text {in }}$ at $1 \mathrm{GHz}$. Inset: Schematic of the measurement setup for RF detection. Losses in measurement cables have been compensated for a precise determination of $\beta$.

frequency range, up to $50 \mathrm{GHz}$. The $45 \mathrm{~nm}$-wide NW-FERs showed a large and flat $\beta$ over almost the entire measured range, corresponding to an improved frequency performance compared to other lateral FERs [24] together with a very good responsivity. The oscillations seen in Fig. 4a are due to impedance mismatches between the devices and the VNA.

The S-parameters of $45 \mathrm{~nm}$-wide NW-FERs were measured (Fig. 4b) to extract the equivalent circuit parameters (inset of $4 b$ ) defining the impedance matrix shown in Eq. 1:

$$
Z_{\mathrm{DUT}}=\left[\begin{array}{cc}
Z_{\mathrm{FER}} & -Z_{\mathrm{FER}} \\
-Z_{\mathrm{FER}} & Z_{\mathrm{FER}}
\end{array}\right] ; Z_{\mathrm{FER}}=R_{\mathrm{s}}+R_{\mathrm{j}} / /\left(j \omega C_{\mathrm{j}}\right)
$$

The fluctuations observed in $S_{21}$ at low frequency are due to the higher noise in the VNA at low frequency and the very low power transmitted through the device. The equivalent circuit element extraction, after open-short de-embedding, resulted in an $R_{\mathrm{s}}$ of $37.9 \pm 1.6 \Omega$, a junction resistance $R_{\mathrm{j}}$ of $101 \pm 17.5$ $\mathrm{k} \Omega$ (similar to the DC $R_{\mathrm{d}}=R_{\mathrm{s}}+R_{\mathrm{j}} \sim R_{\mathrm{j}}$ since $R_{\mathrm{j}} \gg$ $R_{\mathrm{s}}$ ) and junction capacitance $C_{\mathrm{j}}$ of $30.6 \pm 1.7 \mathrm{fF}$ for the 45 $\mathrm{nm}$-wide NW-FERs. S-parameter simulations performed using these parameters show a very good match with the measured ones (Fig. 4.b). In addition, we use these circuit parameters to determine the cut-off frequency, defined as $f_{\mathrm{c}}=1 /\left(2 \pi C_{\mathrm{j}} R_{\mathrm{s}}\right)$ [16], obtaining a value of $140 \mathrm{GHz}$. The cut-off frequency can be significantly improved by reducing $R_{\mathrm{s}}$ and $C_{\mathrm{j}}$ with an optimized geometry, smaller anode and cathode access regions and reduced contact resistances.

The characterization of zero-bias rectifiers also required considerations about the thermal noise generated and rectified by the device itself. This is described by the noise-equivalent power (NEP), which considers the amount of self-generated rectified thermal noise per unit of frequency that produces a DC value at the output. NEP is equal to $\sqrt{4 k_{\mathrm{B}} T R_{0}} / \beta$, where $R_{0}$ is the DC resistance of the device at zero bias. Differently from other reports [20], [36], we believe that the correct value for $R_{0}$ should be $R_{\mathrm{s}}+R_{\mathrm{j}}$ obtained from the $\mathrm{S}$ -

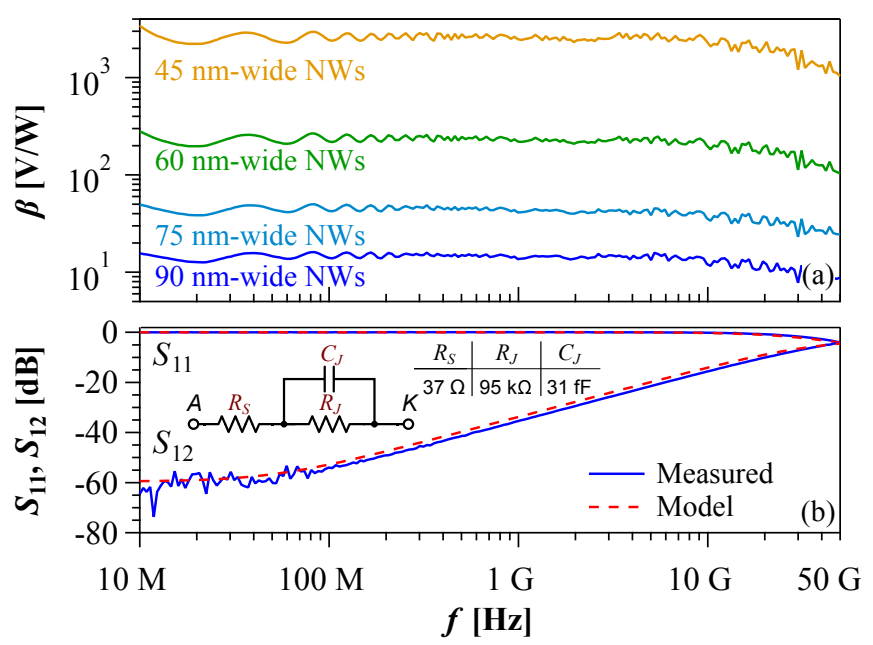

Fig. 4. (a) $\beta$ versus input signal frequency at $P_{\text {in }}=-15 \mathrm{dBm}$. (b) Measured (solid blue) and modeled (dashed red) $S_{11}$ and $S_{12}$ of a 45 nm-wide NWFER. Inset: Equivalent circuit model of the device with values obtained from S-parameter measurement fitting.

parameters, since it represents the real resistance that generates thermal noise when the device is working at a certain power and frequency. With this definition, a NEP of $18.7 \pm 2.3$ $\mathrm{pW} / \sqrt{\mathrm{Hz}}$ was obtained at $1 \mathrm{GHz}$ for $P_{\text {in }}=-20 \mathrm{dBm}$, which is more than $30 \%$ smaller than that for devices with 120 NWs $(\sim 27 \mathrm{pW} / \sqrt{\mathrm{Hz}})$, showing that increasing the number of parallel NWs, thus reducing the device resistance, can effectively reduce the effect of thermal noise.

\section{CONCLUSIONS}

In conclusion, we demonstrated high-performance zero-bias RF rectifiers based on NW-FERs fabricated on a commercial $\mathrm{AlGaN} / \mathrm{GaN}$ HEMT. The use of NWs offered a flexible design tool and much-improved rectification capabilities. By adjusting the width of NWs, $V_{\text {th }}$ could be properly designed, with very high reproducibility, which resulted in a normally-off channel with low $V_{\text {on }}$ (required for zero-bias operation in FERs). The tri-gate structure wrapping the NWs yielded a much better channel control than in planar FERs, resulting in a curvature coefficient of $30.1 \mathrm{~V}^{-1}$, which is close to the theoretical limit of $38.7 \mathrm{~V}^{-1}$. NW-FERs showed a large responsivity of $3000 \mathrm{~V} / \mathrm{W}$, as predicted by the used model, with a flat frequency response, and a high $f_{\mathrm{c}}$ of $140 \mathrm{GHz}$. Such value can be significantly improved by reducing $R_{\mathrm{s}}$ and $C_{\mathrm{j}}$ with an optimized geometry and which opens opportunities for highfrequency detectors. The large responsivity reported together with the good frequency response shows that NW-FERs are an excellent alternative to GaN-HEMT-based SSDs and lateral FERs, thus further expanding the device portfolio compatible with GaN MMICs.

\section{ACKNOWLEDGMENT}

The authors thank the cleanroom staff in the Centre of Micronanotechnology (CMi) and Institute of Physics (IPHYS) at EPFL for technical support. This work was supported by the Swiss National Science Foundation under Grant 200021_169362. 


\section{REFERENCES}

[1] A. Kolpin, S. Winter, R. Weigel, and T. Eireiner, "A sixport receiver's analog front-end for the K-band based on a rapid-prototyped PCB," in MELECON 2006 - 2006 IEEE Mediterranean Electrotechnical Conference, May 2006, pp. 360-362.

[2] J.-R. Yang and S. Hong, "A 24-GHz radar sensor with a six-port network for short-range detection," Microwave and Optical Technology Letters, vol. 56, no. 11, pp. 2634-2637, 2014. [Online]. Available: https://onlinelibrary.wiley.com/doi/abs/10.1002/mop.28665

[3] H. L. Lee, S. Moon, M. Lee, and J. W. Yu, "Six-port QPSK demodulator for optimal K-band multiport amplifier calibration," Electronics Letters, vol. 50, no. 8, pp. 617-618, Apr. 2014.

[4] K. Y. Xu, Y. N. Wang, C. J. Zheng, J. W. Xiong, and G. Wang, "Terahertz Performance of a GaN-Based Planar Nanochannel Device," Journal of Nanomaterials, vol. 2014, p. e850915, Apr. 2014. [Online]. Available: http://www.hindawi.com/journals/jnm/2014/850915/abs/

[5] S.-P. Han, H. Ko, J.-W. Park, N. Kim, Y.-J. Yoon, J.-H. Shin, D. Y. Kim, D. H. Lee, and K. H. Park, "InGaAs Schottky barrier diode array detector for a real-time compact terahertz line scanner," Optics Express, vol. 21, no. 22, p. 25874, Nov. 2013. [Online]. Available: https://www.osapublishing.org/oe/abstract.cfm?uri=oe-21-22-25874

[6] C. Balocco, Y. Pan, S. R. Kasjoo, Y. Alimi, L. Q. Zhang, and A. M. Song, "THz imaging with broadband thermal sources," in 2014 39th International Conference on Infrared, Millimeter, and Terahertz waves (IRMMW-THz), Sep. 2014, pp. 1-2.

[7] A. Tessmann, A. Leuther, H. Massler, S. Wagner, F. Thome, M. Schlechtweg, and O. Ambacher, "A millimeter-wave low-noise amplifier MMIC with integrated power detector and gain control functionality," in 2016 IEEE MTT-S International Microwave Symposium (IMS), May 2016, pp. 1-3.

[8] B. Sahu and G. Rincon-Mora, "A High-Efficiency Linear RF Power Amplifier With a Power-Tracking Dynamically Adaptive Buck-Boost Supply," IEEE Transactions on Microwave Theory and Techniques, vol. 52, no. 1, pp. 112-120, Jan. 2004. [Online]. Available: http://ieeexplore.iee.org/document/1262682/

[9] K. A. Lee, D. H. Lee, J. Park, H.-m. Yoo, and S.-H. Cheon, "A InGaP / GaAs HBT WLA with Power Detector," 2004.

[10] Q. Zhou, K. Wong, W. Chen, and K. J. Chen, "Wide-Dynamic-Range Zero-Bias Microwave Detector Using AlGaN/GaN Heterojunction FieldEffect Diode," IEEE Microwave and Wireless Components Letters, vol. 20, no. 5, pp. 277-279, May 2010.

[11] L. Liu, J. L. Hesler, H. Xu, A. W. Lichtenberger, and R. M. Weikle, "A Broadband Quasi-Optical Terahertz Detector Utilizing a Zero Bias Schottky Diode," IEEE Microwave and Wireless Components Letters, vol. 20, no. 9, pp. 504-506, Sep. 2010.

[12] J. L. Hesler and T. W. Crowe, Responsivity and Noise Measurements of Zero-Bias Schottky Diode Detectors.

[13] P. Chahal, F. Morris, and G. Frazier, "Zero bias resonant tunnel Schottky contact diode for wide-band direct detection," IEEE Electron Device Letters, vol. 26, no. 12, pp. 894-896, Dec. 2005.

[14] N. Su, Z. Zhang, R. Rajavel, P. Deelman, J. N. Schulman, and P. Fay, "Scaling of High-Performance InAs/AlSb/GaSb Heterostructure Detectors for Millimeter-Wave and Submillimeter-Wave Sensing and Imaging," in 2008 Device Research Conference, Jun. 2008, pp. 123124.

[15] H. P. Moyer, J. N. Schulman, J. J. Lynch, J. H. Schaffner, M. Sokolich, Y. Royter, R. L. Bowen, C. F. McGuire, M. Hu, and A. Schmitz, "WBand Sb-Diode Detector MMICs for Passive Millimeter Wave Imaging," IEEE Microwave and Wireless Components Letters, vol. 18, no. 10, pp. 686-688, Oct. 2008.

[16] Z. Zhang, R. Rajavel, P. Deelman, and P. Fay, "Sub-Micron Area Heterojunction Backward Diode Millimeter-Wave Detectors With 0.18 pW/Hz'1/2 Noise Equivalent Power," IEEE Microwave and Wireless Components Letters, vol. 21, no. 5, pp. 267-269, May 2011.

[17] M. Patrashin, N. Sekine, A. Kasamatsu, I. Watanabe, I. Hosako, T. Takahashi, M. Sato, Y. Nakasha, and N. Hara, "GaAsSb/InAlAs/InGaAs Tunnel Diodes for Millimeter Wave Detection in 220330-GHz Band," IEEE Transactions on Electron Devices, vol. 62, no. 3, pp. 1068-1071, Mar. 2015.

[18] S. M. Rahman, Z. Jiang, M. I. B. Shams, P. Fay, and L. Liu, "A GBand Monolithically Integrated Quasi-Optical Zero-Bias Detector Based on Heterostructure Backward Diodes Using Submicrometer Airbridges," IEEE Transactions on Microwave Theory and Techniques, vol. 66, no. 4, pp. 2010-2017, Apr. 2018.
[19] A. M. Song, M. Missous, P. Omling, A. R. Peaker, L. Samuelson, and W. Seifert, "Unidirectional electron flow in a nanometer-scale semiconductor channel: A self-switching device," Applied Physics Letters, vol. 83, no. 9, p. 1881, 2003. [Online]. Available: http://scitation.aip.org/content/aip/journal/apl/83/9/10.1063/1.1606881

[20] P. Sangar, G. Ducournau, B. Grimbert, V. Brandli, M. Faucher, C. Gaquire, A. iguez-de-la Torre, I. iguez-de-la Torre, J. F. Millithaler, J. Mateos, and T. Gonzlez, "Experimental demonstration of direct terahertz detection at room-temperature in $\mathrm{AlGaN} / \mathrm{GaN}$ asymmetric nanochannels," Journal of Applied Physics, vol. 113, no. 3, p. 034305, Jan. 2013. [Online]. Available: http://aip.scitation.org/doi/10.1063/1. 4775406

[21] C. Daher, J. Torres, I. Iiguez-de-la Torre, P. Nouvel, L. Varani, P. Sangar, G. Ducournau, C. Gaquire, J. Mateos, and T. Gonzlez, "Room Temperature Direct and Heterodyne Detection of 0.280.69-THz Waves Based on GaN 2-DEG Unipolar Nanochannels," IEEE Transactions on Electron Devices, vol. 63, no. 1, pp. 353-359, Jan. 2016.

[22] K. Takatani, T. Nozawa, T. Oka, H. Kawamura, and K. Sakuno, "AlGaN/GaN Schottky-ohmic combined anode field effect diode with fluoride-based plasma treatment," Electronics Letters; Stevenage, vol. 44, no. 4, pp. 1-2, Feb. 2008. [Online]. Available: https://search. proquest.com/docview/1625956443/abstract/6CDCC3888DE4DB4PQ/1

[23] Q. Zhou, W. Chen, C. Zhou, B. Zhang, and K. J. Chen, "High sensitivity AlGaN/GaN lateral field-effect rectifier for zero-bias microwave detection," Electronics Letters, vol. 49, no. 22, pp. 1391-1393, Oct. 2013.

[24] K. Wong, W. Chen, Q. Zhou, and K. J. Chen, "Zero-Bias Mixer Based on $\mathrm{AlGaN} / \mathrm{GaN}$ Lateral Field-Effect Diodes for High-Temperature Wireless Sensor and RFID Applications," IEEE Transactions on Electron Devices, vol. 56, no. 12, pp. 2888-2894, Dec. 2009.

[25] W. Chen, K.-Y. Wong, W. Huang, and K. J. Chen, "Highperformance $\mathrm{AlGaNGaN}$ lateral field-effect rectifiers compatible with high electron mobility transistors," Applied Physics Letters, vol. 92, no. 25, p. 253501, Jun. 2008. [Online]. Available: http://aip.scitation.org/doi/10.1063/1.2951615

[26] B. Lu, E. Matioli, and T. Palacios, "Tri-Gate Normally-Off GaN Power MISFET," IEEE Electron Device Letters, vol. 33, no. 3, pp. 360-362, Mar. 2012.

[27] R. G. Meyers, P. Fay, J. N. Schulman, S. Thomas, D. H. Chow, J. Zinck, Y. K. Boegeman, and P. Deelman, "Bias and temperature dependence of Sb-based heterostructure millimeter-wave detectors with improved sensitivity," IEEE Electron Device Letters, vol. 25, no. 1, pp. 4-6, Jan. 2004.

[28] S. Murad, "Dry etching damage in IIIV semiconductors," Journal of Vacuum Science \& Technology B: Microelectronics and Nanometer Structures, vol. 14, no. 6, p. 3658, Nov. 1996. [Online]. Available: http://scitation.aip.org/content/avs/journal/jvstb/14/6/10.1116/1.588745

[29] J. Ma and E. Matioli, "Slanted Tri-Gates for High-Voltage GaN Power Devices," IEEE Electron Device Letters, vol. 38, no. 9, pp. 1305-1308, Sep. 2017

[30] G. Santoruvo and E. Matioli, "In-Plane-Gate GaN Transistors for HighPower RF Applications," IEEE Electron Device Letters, vol. 38, no. 10, pp. 1413-1416, Oct. 2017.

[31] Y. Jo, D. Son, D. Lee, C. Won, J. H. Seo, I. M. Kang, and J. Lee, "First demonstration of GaN-based vertical nanowire FET with topdown approach," in 2015 73rd Annual Device Research Conference (DRC), Jun. 2015, pp. 35-36.

[32] Y. W. Jo, D. H. Son, C. H. Won, K. S. Im, J. H. Seo, I. M. Kang, and J. H. Lee, "AlGaN/GaN FinFET With Extremely Broad Transconductance by Side-Wall Wet Etch," IEEE Electron Device Letters, vol. 36, no. 10, pp. $1008-1010$, Oct. 2015.

[33] A. Westlund, "Self-Switching Diodes for Zero-Bias Terahertz Detection," Ph.D. dissertation, Chalmers University of Technology, Mar. 2015.

[34] Agilent-Technologies, "Square Law and Linear Detection," Nov. 1999. [Online]. Available: http://g3ynh.info/circuits/diode_data/AN986.pdf

[35] X. Zhang, J. Grajal, J. L. Vazquez-Roy, U. Radhakrishna, X. Wang, W. Chern, L. Zhou, Y. Lin, P.-C. Shen, X. Ji, X. Ling, A. Zubair, Y. Zhang, H. Wang, M. Dubey, J. Kong, M. Dresselhaus, and T. Palacios, "Two-dimensional MoS2-enabled flexible rectenna for Wi-Fi-band wireless energy harvesting," Nature, Jan. 2019. [Online]. Available: http://www.nature.com/articles/s41586-019-0892-1

[36] A. Westlund, P. Sangar, G. Ducournau, I. Iiguez-de-la Torre, P.Nilsson, C. Gaquire, L. Desplanque, X. Wallart, J. Millithaler, T. Gonzlez, J. Mateos, and J. Grahn, "Optimization and small-signal modeling of zero-bias InAs self-switching diode detectors," Solid-State Electronics, vol. 104, pp. 79-85, Feb. 2015. [Online]. Available: http://linkinghub.elsevier.com/retrieve/pii/S0038110114002822 